\title{
SOME RESULTS CONCERNING QUASIPERFECT NUMBERS
}

\section{PETER HAGIS, JR. and GRAEME L. COHEN}

(Received June 3, 1981)

Communicated by A. J. van der Poorten

\begin{abstract}
New methods are introduced here to show that if $n$ is a quasiperfect number and $\omega(n)$ the number of its distinct prime factors, then $\omega(n) \geqslant 7$ and $n>10^{35}$, and if further $3 \nmid n$ then $\omega(n) \geqslant 9$ and $n>10^{40}$.

1980 Mathematics subject classification (Amer. Math. Soc.): 10 A 20.
\end{abstract}

\section{Introduction}

A positive integer $n$ is said to be quasiperfect $(Q P)$ if

$$
\sigma(n)=2 n+1
$$

where, as usual, $\sigma(n)$ is the sum of the positive divisors of $n$. Although, as yet, no quasiperfect numbers have been found, several papers have been devoted to a study of the properties which must be possessed by such numbers (if any exist). In the sequel $n$ will always denote a quasiperfect number and $\omega(n)$ will denote the number of distinct prime factors of $n$. The following facts have been previously established:
(A) $n=k^{2}$ where $k$ is odd;
(B) if $m$ is a proper divisor of $n$ then $\sigma(m)<2 m$;
(C) if $r \mid \sigma(n)$ then $r \equiv 1$ or $3(\bmod 8)$;
(D) $\omega(n) \geqslant 6$;

C Copyright Australian Mathematical Society 1982 
(E) if $3 \nmid n$ then $\omega(n) \geqslant 8$;

(F) $n>10^{20}$.

(A), (B) and (C) (implicitly) were proved by Cattaneo (1951), (D) is due to Kishore (1978), and (E) and (F) were proved by Abbott and others (1973).

A result of Dickson (1913) implies that for each $t$ there are only finitely many $Q P$ numbers $n$ with $\omega(n) \leqslant t$. As pointed out by Pomerance (1975), the method of Dickson's proof shows this set is effectively computable.

In the present paper we improve (D), (E) and (F) by proving the following theorems.

THEOREM 1. If $n$ is a quasiperfect number and $(15, n)=1$, then $\omega(n) \geqslant 15$ and $n>10^{57}$.

THeOREM 2. If $n$ is $Q P$ and $3 \nmid n$ then $\omega(n) \geqslant 9$ and $n>10^{40}$.

THEOREM 3. If $n$ is $Q P$ then $\omega(n) \geqslant 7$.

THEOREM 4. If $n$ is $Q P$ then $n>10^{35}$.

\section{Feasible components}

In what follows $p$ and $q$, with or without subscripts, will always denote odd primes. With $(C)$ in mind, and recalling that $\sigma(n)$ is multiplicative, we shall say that " $p^{a}$ is feasible" or "the exponent $a$ is feasible for $p$ " if $2 \mid a$ and if no prime factor of $\sigma\left(p^{a}\right)$ is congruent to 5 or 7 modulo 8 . More generally, $\Pi_{i=1}^{s} p_{i}^{a_{i}}$ will be said to be feasible if $a_{i}$ is feasible for $p_{i}$ for $1 \leqslant i \leqslant s$. If $p^{a} \| n$ then $p^{a}$ must be feasible.

Using the CDC CYBER 174 at the Temple University Computing Center, a search was made for feasible exponents of the odd primes less than 200 . The results appear in Table 1 in Section 8 . In this table, $\beta=\beta(p)$ is the smallest exponent whose feasibility for $p$ was not tested. A lower bound $V$ for $p^{\beta}$ is given for each prime $p$ in Table 1 and, in parentheses next to each feasible exponent $a$ of $p$, the prime factors less than 100 of $\sigma\left(p^{a}\right)$ are listed. For example, $\beta(89)=16$, and if $89^{a} \| n$ and $89^{a}<10^{31}$ then $a=2,8$ or 10 ; also, $67 \mid \sigma\left(89^{10}\right)$ so $a \neq 10$ if $67 \mid n$. For $p=19,29,37, \ldots$, there is no feasible exponent less than $\beta(p)$.

It will be convenient to define $\beta=\beta(p)$ for each odd prime $p$ as follows:

Definition 1. If $p<200$ then the value of $\beta(p)$ is given in Table 1. If $200<p<10^{3}$, then $\beta=8$; if $10^{3}<p<10^{4}$, then $\beta=6$; if $p>10^{4}$, then $\beta=4$. 
It follows that

$$
p^{-\beta-1}<10^{-20}
$$

for every odd prime $p$.

We shall write $\beta_{i}$ for $\beta\left(p_{i}\right)$.

A search was also made for all primes between 200 and 2000 for which $p^{2}$ is feasible. List 1 consists of all such primes for which $p \equiv 2(\bmod 3)$, and List 2 consists of all such primes for which $p \equiv 1(\bmod 3)$. Further, List 3 contains all primes such that $p^{4}$ is feasible where $200<p<2000$. In each list (given in Section 8) all $q$ such that $q \mid \sigma\left(p^{a}\right)$ and $11 \leqslant q<100$ are enclosed in parentheses next to $p$.

REMARK 1. We did not check $p^{a}$ for feasibility if either $p>2000$ or if $a \geqslant 6$ and $200<p<2000$. In what follows, if the feasibility of $p^{a}$ is unknown to us we will treat $p^{a}$ as if it were feasible whenever $2 \mid a$ and $a \leqslant \beta(p)$ (see Definition 1). We note, however, that if $3 \mid n$ and $p \equiv 1(\bmod 3)$ then we cannot have $p^{2} \| n$, since (from (1)) $n$ and $\sigma\left(p^{a}\right)$ are relatively prime whenever $p^{a} \| n$.

REMARK 2. Our lists considerably extend (and amend) lists in Abbott and others (1973), Lemma 4A. The lists given there were constructed on the basis of congruence arguments (for example, $p^{2} \nVdash n$ when $p \equiv 2$ or $4(\bmod 7)$ ). More information than we require here is contained in our lists, but they should prove useful to others working with quasiperfect numbers.

\section{Two important lemmas}

Our next objective is to prove two lemmas which will be very useful in the remaining sections of this paper. For convenience we first introduce the notation $h(k)=\sigma(k) / k$, where $k$ is a positive integer. $h(k)$ is multiplicative, $h\left(p^{\infty}\right)=$ $\lim _{a \rightarrow \infty} h\left(p^{a}\right)=p /(p-1)$; and it is easy to see that $1 \leqslant h\left(p^{a}\right)<h\left(p^{b}\right)$ if $0 \leqslant a<b \leqslant \infty$, and $h\left(p^{a}\right)<h\left(q^{b}\right)$ if $p>q$ and $0 \leqslant a \leqslant \infty, 1 \leqslant b \leqslant \infty$. (See Section 1.3 in Pomerance (1974).)

From (1) and (F) we see that

$$
2<h(n)<2+10^{-20}=2^{*},
$$

from which it follows that

$$
\prod_{p \mid n} p /(p-1)=\prod_{p \mid n} h\left(p^{\infty}\right)>2 \text { if } n \text { is } Q P .
$$


Lemma 1. Suppose that $n$ is $Q P$ and $n=\prod_{i=1}^{s} p_{i}^{a_{i}} \prod_{j=1}^{t} q_{j}^{b_{j}}=M N$ where $(M, N)$ $=1, s \geqslant 1, t \geqslant 1$. Let $B=2^{*} / h\left(M^{\prime}\right)$ where $M^{\prime}=\prod_{i=1}^{s} p_{i}^{c_{i}}$ with $2 \leqslant c_{i} \leqslant a_{i}$, and let $D=h\left(M^{\prime \prime}\right) / 2$ where $M^{\prime \prime}=\prod_{i=1}^{s} p_{i}^{d_{i}}$ with $a_{i} \leqslant d_{i} \leqslant \infty$. Suppose also that $q_{1}<q_{2}<\cdots<q_{r}$. Then:

$$
\begin{gathered}
q_{1}>(\sqrt{4 B-3}+1) / 2(B-1), \\
q_{1}<\left(1-D^{1 / t}\right)^{-1} .
\end{gathered}
$$

Proof. Suppose that $q_{1} \leqslant(\sqrt{4 B-3}+1) / 2(B-1)$. Then it is an algebraic exercise to verify that $\left(1 / q_{1}+1 / 2\right)^{2} \geqslant B-3 / 4$ so that $1+q_{1}^{-1}+q_{1}^{-2} \geqslant B$. Using (3) and (A) we see that

$$
\begin{aligned}
2^{*} & >h(n)=h(M) \cdot h(N) \geqslant h\left(M^{\prime}\right) \cdot h\left(q_{1}^{2}\right) \\
& =2^{*} B^{-1}\left(1+q_{1}^{-1}+q_{1}^{-2}\right) \geqslant 2^{*} B^{-1} B=2^{*} .
\end{aligned}
$$

This contradiction proves (i). Now assume that $q_{1} \geqslant\left(1-D^{1 / t}\right)^{-1}$. Then $q_{1} /\left(q_{1}-1\right) \leqslant D^{-1 / t}$ so that

$$
\begin{aligned}
2 & <h(n)=h(M) \cdot h(N)<h(M) \prod_{j=1}^{t} q_{j} /\left(q_{j}-1\right) \\
& \leqslant h\left(M^{\prime \prime}\right) \cdot\left(q_{1} /\left(q_{1}-1\right)\right)^{t} \leqslant h\left(M^{\prime \prime}\right)\left(D^{-1 / t}\right)^{t}=2 D D^{-1}=2 .
\end{aligned}
$$

This contradiction proves (ii).

Lemma 2. Suppose $n$ is $Q P$, and write $n=N p^{a}$, where $p \nmid N$. Write also

$$
\begin{gathered}
N=M \prod_{j=1}^{t} q_{j}^{b_{j}} \text { where } q_{j} \nmid M, \\
F \risingdotseq h(M) \prod_{j=1}^{t} q_{j}\left(q_{j}-1\right), \quad E=\sum_{j=1}^{t} q_{j}^{-b_{j}-1}, \\
R=2 /(2-F), \quad \text { and } \\
L=R-2 F U /(2-F)(2-F+F U),
\end{gathered}
$$

where $U$ is any upper bound for $E$. If $F<2$, then

$$
L-p^{-1} \leqslant p<R \text {. }
$$

Further, if $q<p$ are the two largest prime factors of $n$, then

$$
L^{\prime}=L-\left(L-(q+2)^{-1}\right)^{-1} \leqslant p<R .
$$

Proof. According to Jerrard and Temperley (1973), Proposition 3,

$$
2 /(2-h(N))-p^{-1} \leqslant p<2 /(2-h(N)),
$$


and, as shown by Pomerance (1974), Section 1.5,

$$
F-F E \leqslant h(N) \leqslant F \text {. }
$$

Therefore, when $F<2$,

$$
\begin{aligned}
2 /(2-F)-2 /(2-h(N)) & \leqslant 2 /(2-F)-2 /(2-F+F E) \\
& =2 F E /(2-F)(2-F+F E) \\
& \leqslant 2 F U /(2-F)(2-F+F U),
\end{aligned}
$$

since $E \leqslant U$ and $x /(c+x)$ is monotonic increasing when $c>0$, and (8) follows. Then $p \geqslant L-(q+2)^{-1}$ and (9) also follows.

\section{The proof of Theorem 1}

$$
\text { If }(n, 15)=1 \text { and } \omega(n) \leqslant 14 \text { then, since } h\left(p^{a}\right)<h\left(p^{\infty}\right)=p /(p-1) \text {, }
$$

$$
h(n)<\prod_{p \mid n} p /(p-1) \leqslant \prod_{p=7}^{59} p /(p-1)<2 .
$$

Hence, $\omega(n) \geqslant 15$; and $n \geqslant(7 \cdot 17 \cdot 31 \cdot 41 \cdot 71 \cdot 73 \cdot 89 \cdot 97 \cdot 103 \cdot 127 \cdot 167$. $199 \cdot 223 \cdot 239 \cdot 241)^{2}>10^{57}$, since $13^{6}>23^{4}>241^{2}$. (See Table 1 and Lists 1, 2,3.)

\section{The proof of Theorem 2}

We shall prove here only the second part of the theorem, since our method for showing that $\omega(n) \geqslant 9$ when $3 \nmid n$ is similar to that used in the proof of Theorem 3 , and requires less computing. Full details of the proof are available from either of the authors.

By Theorem 1, if $(15, n)=1$ then $n>10^{57}$. Therefore, in the remainder of this section we assume that $3 \nmid n$ and $5 \mid n$. If $n$ is divisible by 11,19 or 29 then (see Table 1) $n \geqslant 5^{6} \cdot 11^{16}(7 \cdot 17 \cdot 31 \cdot 41 \cdot 71 \cdot 73 \cdot 89)^{2}>10^{42}$. Therefore, we may assume that $(11 \cdot 19 \cdot 29, n)=1$. If $13 \nmid n$ then $\omega(n) \geqslant 14$ since $\Pi_{p=5}^{67} p /(p-1)$ $\cdot(10 / 11)(12 / 13)(18 / 19)(28 / 29)<2$; and if $13 \mid n$ then $\omega(n) \geqslant 11$ since $\Pi_{p=5}^{47} p /(p-1) \cdot(10 / 11)(18 / 19)(28 / 29)<2$. It follows easily that $n>5^{6}$. $13^{6}(7 \cdot 17 \cdot 31 \cdot 41 \cdot 71 \cdot 73 \cdot 89 \cdot 97 \cdot 103)^{2}>10^{40}$.

\section{The proof of Theorem 3}

By (D) we need only prove that $\omega(n) \neq 6$. We suppose, therefore, that $n=\Pi_{i=1}^{6} p_{i}^{a_{i}}$ where $p_{1}<p_{2}<\cdots<p_{6}$. By Theorem 2, $p_{1}=3$ and since $(3 / 2) \prod_{p=13}^{29} p /(p-1)<2$ we see that $5 \leqslant p_{2} \leqslant 11$. If $p_{2}=5$ then, by Lemma 1 
with $M^{\prime}=3^{4} 5^{6}$, we have $p_{3} \geqslant 17$; and since $(3 / 2)(5 / 4) \prod_{p=59}^{71} p /(p-1)<2$ we see that $p_{3} \leqslant 53$. If $p_{2}=7$ then, since $(3 / 2)(7 / 6) \prod_{p=29}^{41} p /(p-1)<2$, we see that $11 \leqslant p_{3} \leqslant 23$. If $p_{2}=11$ then $13 \leqslant p_{3} \leqslant 17$ since

$$
(3 / 2)(11 / 10) \prod_{p=19}^{31} p /(p-1)<2 \text {. }
$$

A computer program utilizing double precision arithmetic was written which for each feasible value of $M=p_{1}^{a_{1}} p_{2}^{a_{2}} p_{3}^{a_{3}}$ used Lemma 1 to find upper and lower bounds for $p_{4}$. For $a_{i}<\beta_{i}$ (see Definition 1 and Table 1) we took $c_{i}=a_{i}$ in $M^{\prime}$ and $d_{i}=a_{i}$ in $M^{\prime \prime}$, while for $a_{i} \geqslant \beta_{i}$ we took $c_{i}=\beta_{i}$ in $M^{\prime}$ and $d_{i}=\infty$ in $M^{\prime \prime}$. Thus, the infinite set of $M$ 's was investigated by examining the elements of a finite set. For example, it was found that $127 \leqslant p_{4} \leqslant 761$ for $p_{1}=3, p_{2}=5$, $p_{3}=17$. The program then used Lemma 1 to find bounds on $p_{5}$ for each feasible value of $M=p_{1}^{a_{1}} p_{2}^{a_{2}} p_{3}^{a_{3}} p_{4}^{a_{4}}$. (We included $p_{4}^{6}$ and $p_{4}^{8}$ as possible factors of $M$ but did not check for feasibility if $p_{4}>200$. See Remark 1 . No $p_{4}$ exceeded 761.)

Since $n=\Pi_{i=1}^{6} p_{i}^{a_{i}}$ we can write (see (5)) $n=p_{6}^{a_{6}} N=p_{6}^{a_{6}} M \Pi_{j=1}^{t} q_{j}^{b_{j}}$ where $p_{i} \mid M$ if $a_{i}<\beta_{i}$ and $p_{i}=q_{j}$ for some $j$ if $a_{i} \geqslant \beta_{i}$. From (2), $E=\Sigma_{j=1}^{t} q_{j}^{-b_{j}-1}<t$. $10^{-20} \leqslant 5 \cdot 10^{-20}=U$. The program calculated $F$ (which was always less than 2 ), as given by (6), for all possible values of $M$ and $q_{1}, \ldots, q_{t}$ as determined by the values of $p_{1}, p_{2}, \ldots, p_{5}$ previously found and the exponents implied by Definition 1 and Remark 1. (For example, if $p_{5}>10000$ and $p_{5} \equiv 2(\bmod 3)$ then either $p_{5}^{2} \| M$ or $p_{5}=q_{t}$.) Also, $R$ and $L^{\prime}$ (with $q=p_{5}$ ), as defined in (7) and (9), were calculated in order to determine $p_{6}$. For only 12 quintets $\left(p_{1}, p_{2}, p_{3}, p_{4}, p_{5}\right)$ did $R$ and $L^{\prime}$ span a prime, thus yielding a (possible) value of $p_{6}$. The (possible) prime factors of a six component quasiperfect number are given in Table 2.

TABLE 2

\begin{tabular}{|c|c|c|c|c|c|}
\hline$p_{1}$ & $p_{2}$ & $p_{3}$ & $p_{4}$ & $p_{5}$ & $p_{6}$ \\
\hline 3 & 5 & 17 & 257 & 65537 & 4294427569 \\
3 & 5 & 17 & 337 & 1709 & 1783 \\
3 & 5 & 17 & 571 & 743 & 983 \\
3 & 5 & 19 & 151 & 503 & 541 \\
3 & 5 & 19 & 197 & 347 & 401 \\
3 & 5 & 23 & 59 & 193 & 521 \\
3 & 5 & 43 & 59 & 67 & 113 \\
3 & 7 & 11 & 37 & 103 & 191 \\
3 & 7 & 11 & 47 & 107 & 137 \\
3 & 7 & 11 & 71 & 79 & 89 \\
3 & 7 & 13 & 41 & 43 & 107 \\
3 & 11 & 13 & 23 & 29 & 31 \\
\hline
\end{tabular}


We now write $n=\prod_{i=1}^{6} p_{i}^{a_{i}}=m \prod_{j=1}^{t} q_{j}^{b_{j}}$ where $p_{i} \mid m$ if $a_{i}<\beta_{i}$ and $p_{i}=q_{j}$ for some $j$ if $a_{i} \geqslant \beta_{i}$. For all possible values of $m$ and $q_{1}, \ldots, q_{t}$ as determined by the values of $p_{i}$ given in Table 2 and the exponents implied by Definition 1 and Remark $1, h(m) \Pi_{j=1}^{t} q_{j} /\left(q_{j}-1\right)$ was calculated and $h(n)$ was then bounded using (10). In every case either $h(n)<2$ or $h(n)>2+10^{-20}$. This contradiction to (3) completes the proof of Theorem 3.

\section{The proof of Theorem 4}

Theorem 4 is proved by establishing the twenty facts below. Only eight of these are proved here; the details of the remaining proofs are available from either of the authors. Notice first that, by Theorem 2 , if $3+n$ then $n>10^{40}$, so that we may assume that $3 / n$. We shall use:

Lemma 3. Suppose that $n$ is $Q P$ and $3 \mid n$. Then there is an even number of components $q^{b}$ of $n$ with $q \equiv b \equiv 1(\bmod 3)$.

Proof. From (1), and since $3 \mid n, \sigma(n) \equiv 1(\bmod 3)$. Then there must be an even number of components $q^{b}$ of $n$ with $\sigma\left(q^{b}\right) \equiv 2(\bmod 3)$. If $q^{b}$ is such a component, then $q \neq 3$ and $q \neq 2(\bmod 3)$ since $b$ is even, by (A). Thus $q \equiv 1$ $(\bmod 3)$, so $\sigma\left(q^{b}\right) \equiv b+1 \equiv 2(\bmod 3)$. Thus $b \equiv 1(\bmod 3)$. This proves the lemma.

I. If $p^{a} \| n$ and $p^{a} \geqslant 11^{16}$ then $n>10^{35}$.

Proof. Since the six smallest possible components of $n$ are $3^{4}<17^{2}<41^{2}<7^{4}$ $<71^{2}<89^{2}$ (see Table 1) it follows that $n \geqslant 3^{4} 7^{4} 11^{16}(17 \cdot 41 \cdot 71 \cdot 89)^{2}>10^{35}$.

II. If $S=\{11,19,29,37,53,59,73,97,101,113,131,137,157,163,173,181$, $191,197,199\}$ and $p \mid n$ where $p \in S$, then $n>10^{35}$. If $43 \cdot 47 \mid n$ then $n>10^{35}$.

ProOf. This follows immediately from I and an examination of Table 1.

III. If $\omega(n) \geqslant 10$ then $n>10^{35}$.

Proof. If $\omega(n) \geqslant 10$ then $n \geqslant 3^{4} 5^{6} 7^{4}(17 \cdot 41 \cdot 71 \cdot 89 \cdot 167 \cdot 239 \cdot 311)^{2}>$ $10^{36}$.

We assume hereafter that $p \notin S$ if $p \mid n$, that $43 \cdot 47 \nmid n$ and that $\omega(n)=7,8$ or 9. 
IV. If $(n, 35)=1$ then $n>10^{35}$.

Proof. Assume that $(n, 35)=1$. If $13 \nmid n$ then, since $\omega(n) \leqslant 9, h(n)<$ $(3 / 2)(17 / 16)(23 / 22)(31 / 30)(41 / 40)(43 / 42)(61 / 60)(67 / 66)(71 / 70)<2$ which contradicts (3). If $13 \mid n$ then $\omega(n)=9$ since

$$
(3 / 2)(13 / 12)(17 / 16)(23 / 22)(31 / 30)(41 / 40)(43 / 42)(61 / 60)<2 \text {, }
$$

so $n \geqslant 3^{4} 13^{6}(17 \cdot 41 \cdot 71 \cdot 89 \cdot 167 \cdot 239 \cdot 311)^{2}>10^{36}$.

We may now assume that $5 \mid n$ or $7 \mid n$. (Notice that $5 \cdot 7 \nmid n$ since $h\left(3^{4} 5^{6} 7^{4}\right)>$ 2*. See Lemma 4B in Abbott and others (1973).)

V. If $3^{28} \mid n$ then $n>10^{35}$.

From Table 1 we may now assume that $3^{4} \| n$ or $3^{12} \| n$.

It will be convenient for what follows to now make the following definition.

DEFINITION 2. The positive integer $s$ will be said to be satisfactory if $s=3^{\alpha} q^{\gamma} \mu^{2}$ where: (i) $\alpha=4$ or 12 ; (ii) $q=5$ or 7 ; (iii) $\mu$ has no prime factor belonging to $S$ or $\{2,3,5,7\}$; (iv) $p \equiv 1(\bmod 3)$ implies that $p^{2} H s$; (v) $\omega(s)=7,8$ or 9 ; (vi) $2<h(s)<2 *$.

We see immediately that if $n$ is $Q P$ and $n<10^{35}$ then $n$ is satisfactory.

VI. If $5^{16} \mid n$ then $n>10^{35}$.

Proof. If $5^{16} \mid n$ then, from Table 1 , either $5^{16} \| n$ or $5^{22} \mid n$. In the latter case $n \geqslant 3^{4} 5^{22}(17 \cdot 41 \cdot 71 \cdot 89 \cdot 167)^{2}>10^{35}$. Now suppose that $3^{12} \| n$. If $17 \nmid n$ then $n \geqslant 3^{12} 5^{16}(41 \cdot 71 \cdot 89 \cdot 167 \cdot 239)^{2}>10^{36}$. If $17 \mid n$ then, by Lemma 1 with $M^{\prime}$ $=3^{12} 5^{16} 17^{2}, p \nmid n$ if $17<p<251$. Therefore, $n \geqslant 3^{12} 5^{16}(17 \cdot 311 \cdot 353 \cdot 383$. $449)^{2}>10^{39}$. We can now assume that $3^{4} 5^{16} \| n$. Since $\sigma\left(3^{4}\right)=121$ and $409 \mid \sigma\left(5^{16}\right)$ we see from $(1)$ that $n \equiv 60(\bmod 121)$ and $n \equiv 204(\bmod 409)$. Let $n=3^{4} 5^{16} x^{2}$ and suppose that $n<10^{35}$. Then it is easy to verify that $53 x^{2} \equiv 60(\bmod 121)$ and $98 x^{2} \equiv 204(\bmod 409)$. These congruences have four positive simultaneous solutions less than $m=121 \cdot 409$, namely, $x_{1}=7343, x_{2}=22105, x_{3}=27384, x_{4}=$ 42146. These were found using the Chinese Remainder Theorem. For $i=1,2,3,4$ and $0 \leqslant k \leqslant\left[10^{17.5} / 3^{2} 5^{8} \mathrm{~m}\right]=1817560$ the integers $n(i, k)=3^{4} 5^{16}\left(x_{i}+k m\right)^{2}$ were decomposed as necessary into their prime factors. No satisfactory values of $n(i, k)$ were found. We conclude that if $3^{4} 5^{16} \| n$ then $n>10^{35}$.

We may now assume, using Table 1 , that $5^{6} \| n$ if $5 \mid n$. 
VII. If $3^{12} 5^{6} \| n$ then $n>10^{35}$.

VIII. If $7^{12} \mid n$ then $n>10^{35}$.

IX. If $7^{10} \| n$ then $n>10^{35}$.

X. If $3^{12} 7^{4} \| n$ then $n>10^{35}$.

We may now assume that either $3^{4} 5^{6} \| n$ or $3^{4} 7^{4} \| n$.

XI. If $23^{10} \mid n$ then $n>10^{35}$.

Proof. If $5 \mid n$ then by Lemma 1 , with $M^{\prime}=3^{4} 5^{6} 23^{10},(17 \cdot 41, n)=1$. Therefore, $n \geqslant 3^{4} 5^{6} 23^{10}(71 \cdot 89 \cdot 167 \cdot 239)^{2}>10^{36}$. If $7^{4} \| n$ it is easy to verify, using Lemma 3 and Table 1 , that $n>10^{35}$ unless $43^{4} \| n$. If $17 \nmid n$ then $n \geqslant$ $3^{4} 7^{4} 23^{10} 43^{4}(41 \cdot 71 \cdot 89)^{2}>10^{36}$. If $17 \mid n$ then by Lemma 1 , with $M^{\prime}=$ $3^{4} 7^{4} 17^{2} 23^{10} 43^{4},(41 \cdot 71 \cdot 89, n)=1$. In that case, $n \geqslant 3^{4} 7^{4} 23^{10} 43^{4}(17 \cdot 167$. $239)^{2}>10^{37}$.

From this and Table 1 we may now assume that if $23 \mid n$ then $23^{4} \| n$.

XII. If $17^{10} \mid n$ then $n>10^{35}$.

XIII. If $5 \cdot 17 \mid n$ then $n>10^{35}$.

Henceforth we may assume that $17 \nmid n$ if $5 \mid n$.

XIV. If $5 p \mid n$, where $p=23,31$ or 43 , then $n>10^{35}$.

Proof. By XI, I and Table 1 we may assume that $3^{4} 5^{6} 23^{4} \| n$ or $3^{4} 5^{6} 31^{6} \| n$ or $3^{4} 5^{6} 43^{4} \| n$. Let $n=3^{4} 5^{6} p^{2 a} x^{2}$ where $p^{2 a}=23^{4}, 31^{6}$ or $43^{4}$ and suppose that $n<10^{35}$. From (1) we see that $86\left(p^{a} x\right)^{2} \equiv 60(\bmod 121)$ and $15641\left(p^{a} x\right)^{2} \equiv$ $9765(\bmod 19531)$. The four common incongruent solutions of these congruences are $X_{1}=264434\left(p^{a}\right)^{-1}, \quad X_{2}=341027\left(p^{a}\right)^{-1}, \quad X_{3}=2022224\left(p^{a}\right)^{-1}, \quad X_{4}=$ 2098817( $\left.p^{a}\right)^{-1}$ where $y^{-1}$ is the reciprocal of $y$ modulo $m=121 \cdot 19531$. For $i=1,2,3,4$ let $x_{i}=x_{i}\left(p^{a}\right)$ be the least positive residue of $X_{i}$ modulo $m$. Also, let $K=K\left(p^{a}\right)=\left[10^{17.5} / 3^{2} 5^{3} p^{a} m\right]$. Then $K\left(23^{2}\right)=224844, K\left(31^{3}\right)=3992$ and $K\left(43^{2}\right)=64328$. For $i=1,2,3,4$ and $0 \leqslant k \leqslant K$ the integers $n\left(i, k, p^{a}\right)=$ $3^{4} 5^{6} p^{2 a}\left(x_{i}+k m\right)^{2}=3^{4} 5^{6} p^{2 a} \mu^{2}$ were decomposed as necessary into their prime factors (unless $p \mid \mu$ ). No satisfactory value of $n\left(i, k, p^{a}\right.$ ) was found. 
XV. If $5 \cdot 41 \mid n$ then $n>10^{35}$.

XVI. If $5 \mid n$ then $n>10^{35}$.

XVII. If $7 \cdot 23 \mid n$ then $n>10^{35}$.

XVIII. If $7 \cdot 13 p \mid n$, where $p=41$ or 43 , then $n>10^{35}$.

XIX. If $7 \cdot 17 p \mid n$, where $p=31$ or 41, then $n>10^{35}$.

$\mathrm{XX}$. If $7 \mid n$ then $n>10^{35}$.

Proof. We may assume that $3^{4} 7^{4} \| n$, and by XVII we may suppose that $23 \nmid n$. Since $h\left(3^{4} 7^{4} 13^{6} 17^{2}\right)>2^{*}$ we see that $13 \cdot 17 \nmid n$. Let $n=3^{4} 7^{4} 13^{c} 17^{d} P^{2}$ where $c=0$ or 6 and $d=0,2$ or 8 . By Lemma $3, q^{2 b} \| n$ where $q>17, q \equiv 1(\bmod 3)$, $b>1$ and $b \neq 3$. If $c=d=0$, then, since

$$
(3 / 2)(7 / 6)(31 / 30)(41 / 40)(43 / 42)(61 / 60)(67 / 66)(71 / 70)<2,
$$

$\omega(n)=9$ and $n \geqslant 3^{4} 7^{4} 43^{4}(41 \cdot 71 \cdot 89 \cdot 167 \cdot 239 \cdot 311)^{2}>10^{36}$. If $17 \mid n$ then by XIX, we may assume that $(31 \cdot 41, n)=1$. Since

$$
(3 / 2)(7 / 6)(17 / 16)(43 / 42)(61 / 60)(67 / 66)(71 / 70)<2
$$

we see that $\omega(n)=8$ or 9 . Since $3^{4} 7^{4} 17^{2}(43 \cdot 67 \cdot 79)^{4}(71 \cdot 89)^{2}>10^{36}$ we may assume that at most two prime factors $q>17$ of $n$ have exponents greater than 2 . If $q^{4} p^{6} \mid n$ where $q$ and $p$ exceed 17 then $n \geqslant 3^{4} 7^{4} 17^{2} 43^{4} 61^{6}(71 \cdot 89 \cdot 167)^{2}>10^{37}$. Otherwise,

$$
\begin{aligned}
h(n)< & (121 / 81)(7 / 6)(17 / 16)(43 / 42)(67 / 66) \\
& \times(71 / 70)(89 / 88)(167 / 166)(239 / 238)<2 .
\end{aligned}
$$

Now suppose that $13 \mid n$. Then, by XVIII, we may assume that $(41 \cdot 43, n)=1$. If $p^{6} \| n$ for $p>13$ then $n \geqslant 3^{4} 7^{4} 13^{6} 31^{6} 67^{4} 71^{2} 89^{2}>10^{35}$. Therefore, we may assume (see I) that $(31 \cdot 47 \cdot 61, n)=1$, and since

$$
(121 / 81)(7 / 6)(13 / 12)(67 / 66)(71 / 70)(79 / 78)(83 / 82)<2
$$

it follows that $\omega(n) \geqslant 8$. Then, $n \geqslant 3^{4} 7^{4} 13^{6} 67^{4}(71 \cdot 89 \cdot 167 \cdot 239)^{2}>10^{36}$.

Theorem 4 now follows from IV, XVI and XX. 


\section{Tables}

\section{TABLE 1}

Feasible exponents $a$ for primes $p<200$ if $p^{u}$ is a component of a quasiperfect number. For each $p, p^{u}<V<p^{\beta}$ where $\beta$ need not be feasible. Prime factors $<100$ of $\sigma\left(p^{\triangleleft}\right)$ are given in parentheses next to $a$. (See Section 2.)

\begin{tabular}{|c|c|c|c|c|c|c|c|}
\hline$p$ & $a$ & $\beta$ & $V$ & $p$ & $a$ & $\beta$ & $V$ \\
\hline 3 & $\begin{array}{r}4(11), 12,28(59) \\
40(83)\end{array}$ & 52 & $10^{24}$ & 5 & $6,16,22$ & 40 & $10^{27}$ \\
\hline 7 & $\begin{array}{r}2(3,19), 4,10 \\
12,16,18\end{array}$ & 28 & $10^{23}$ & 11 & 16 & 28 & $10^{29}$ \\
\hline 13 & 6 & 40 & $10^{44}$ & 17 & $2,8(19), 10$ & 26 & $10^{31}$ \\
\hline 19 & & 16 & $10^{20}$ & 23 & $4,10(11), 12$ & 18 & $10^{24}$ \\
\hline 29 & & 22 & $10^{32}$ & 31 & $2(3), 6$ & 16 & $10^{23}$ \\
\hline 37 & & 30 & $10^{47}$ & 41 & 2 & 16 & $10^{25}$ \\
\hline 43 & 4,12 & 24 & $10^{39}$ & 47 & $6(43), 10$ & 16 & $10^{26}$ \\
\hline 53 & & 30 & $10^{51}$ & 59 & & 12 & $10^{21}$ \\
\hline 61 & 6 & 30 & $10^{53}$ & 67 & 4 & 24 & $10^{43}$ \\
\hline 71 & 2 & 12 & $10^{22}$ & 73 & $2(3)$ & 16 & $10^{29}$ \\
\hline 79 & 4,6 & 18 & $10^{34}$ & 83 & 4 & 12 & $10^{23}$ \\
\hline 89 & $2,8(73), 10(11,67)$ & 16 & $10^{31}$ & 97 & $2(3), 8(3), 10(89)$ & 16 & $10^{31}$ \\
\hline 101 & & 30 & $10^{60}$ & 103 & $2(3), 4(11)$ & 16 & $10^{32}$ \\
\hline 107 & 4 & 12 & $10^{24}$ & 109 & 6 & 16 & $10^{32}$ \\
\hline 113 & & 10 & $10^{20}$ & 127 & $2(3), 4$ & 12 & $10^{25}$ \\
\hline 131 & & 16 & $10^{33}$ & 137 & & 10 & $10^{21}$ \\
\hline 139 & $4(41)$ & 12 & $10^{25}$ & 149 & 6 & 16 & $10^{34}$ \\
\hline 151 & 6 & 12 & $10^{26}$ & 157 & & 16 & $10^{35}$ \\
\hline 163 & & 12 & $10^{26}$ & 167 & 2,8 & 10 & $10^{22}$ \\
\hline 173 & & 16 & $10^{35}$ & 179 & $4(11)$ & 12 & $10^{27}$ \\
\hline 181 & & 16 & $10^{36}$ & 191 & & 10 & $10^{22}$ \\
\hline 193 & & 16 & $10^{36}$ & 197 & & 16 & $10^{36}$ \\
\hline 199 & 2(3) & 10 & $10^{22}$ & & & & \\
\hline
\end{tabular}

List 1. Primes $p$ such that $p^{2}$ is feasible where $p \equiv 2(\bmod 3)$ and $200<p<$ 2000. Prime factors $<100$ of $\sigma\left(p^{2}\right)$ are given in parentheses next to $p$. 239(19), 311(19), 353(19), 383, 449(97), 479(43), 593, 617(97), 647, 743, 761, 839, 857, $881(19), 911,1097,1151(19), 1193,1217,1223(19), 1487,1559,1583,1847$, 1889(73). 
List 2. Primes $p$ such that $p^{2}$ is feasible where $p \equiv 1(\bmod 3)$ and $200<p<$ 2000. Prime factors (other than 3 ) $<100$ of $\sigma\left(p^{2}\right)$ are given in parentheses next to p. 223, 241, 271, 337(43), 409, 463(19), 577(19), 631, 673, 727, 937, 967(67), 1039, 1063, 1249(73), 1447, 1489(19), 1567, 1609, 1657, 1753, 1879, 1993.

LIST 3. Primes $p$ such that $p^{4}$ is feasible where $200<p<2000$. Prime factors $<100$ of $\sigma\left(p^{4}\right)$ are given in parentheses next to $p .227,239,263,359,367(11)$, 379(11,41), 439, 443(11), 463, 479, 503, 523, 563, 587(11), 643(11), 647(11), 719(11), 727, 887, 919, 967, 983(11), 1063, 1103(11,41), 1123(41), 1187, 1223, 1259(11), 1327, 1439(11), 1487, 1499(11), 1579, 1627, 1663, 1699(11,41), 1759(41), 1787(11), 1847, 1879(11), 1979, 1987.

\section{References}

H. L. Abbott, C. E. Aull, Ezra Brown and D. Suryanarayana (1973), 'Quasiperfect numbers', Acta Arith. 22, 439-447; (1976), "Corrections to the paper "Quasiperfect numbers", Acta Arith. 29, $427-428$.

Paolo Cattaneo (1951), 'Sui numeri quasiperfetti’, Boll. Un. Mat. Ital. (3) 6, 59-62.

L. Dickson (1913), 'Finiteness of the odd perfect and primitive abundant numbers with $n$ distinct prime factors', Amer. J. Math. 35, 413-422.

R. P. Jerrard and Nicholas Temperley (1973), 'Almost perfect numbers', Math. Mag. 46, 84-87.

Masao Kishore (1978), 'Odd integers $N$ with five distinct prime factors for which $2-10^{-12}<$ $\sigma(N) / N<2+10^{-12}$, Math. Comp. 32, 303-309.

C. Pomerance (1974), 'Odd perfect numbers are divisible by at least seven distinct primes', Acta Arith . 25, 265-300.

C. Pomerance (1975), 'The second largest prime factor of an odd perfect number', Math. Comp. 29 , 914-921.

Department of Mathematics

Temple University

Philadelphia, Pennsylvania 19122

U.S.A.
School of Mathematical Sciences

The New South Wales Institute

of Technology

Broadway, New South Wales 2007

Australia 